\title{
Measuring Personal Exposure to Organophosphate Flame Retardants using Silicone Wristbands and Hand Wipes
}

\author{
Stephanie C. Hammel ${ }^{\dagger}$, Kate Hoffman $^{\dagger}$, Thomas F. Webster ${ }^{\ddagger}$, Kim A. Anderson ${ }^{\S}$, and \\ Heather M. Stapleton ${ }^{\dagger,}$ * \\ ${ }^{\dagger}$ Nicholas School of the Environment, Duke University, Durham, North Carolina, United States \\ ¥Department of Environmental Health, Boston University School of Public Health, Boston, \\ Massachusetts, United States \\ §Department of Environmental and Molecular Toxicology, Oregon State University, Corvallis, \\ Oregon, United States
}

\begin{abstract}
Organophosphate flame retardants (PFRs) are widely used as replacements for polybrominated diphenyl ethers in consumer products. With high detection in indoor environments and increasing toxicological evidence suggesting a potential for adverse health effects, there is a growing need for reliable exposure metrics to examine individual exposures to PFRs. Silicone wristbands have been used as passive air samplers for quantifying exposure in the general population and occupational exposure to polycyclic aromatic hydrocarbons. Here we investigated the utility of silicone wristbands in measuring exposure and internal dose of PFRs through measurement of urinary metabolite concentrations. Wristbands were also compared to hand wipes as metrics of exposure. Participants wore wristbands for five consecutive days and collected first morning void urine samples on three alternating days. Urine samples were pooled across the three days and analyzed for metabolites of the following PFRs: tris(1,3-dichloroisopropyl) phosphate (TDCIPP), tris(1chloro-2-isopropyl) phosphate (TCIPP), triphenyl phosphate (TPHP), and mono-substituted isopropylated triaryl phosphate (mono-ITP). All four PFRs and their urinary metabolites were ubiquitously detected. Correlations between TDCIPP and TCIPP and their corresponding urinary metabolites were highly significant on the wristbands $\left(\mathrm{r}_{\mathrm{s}}=0.5-0.65, \mathrm{p}<0.001\right)$, which suggest that wristbands can serve as strong predictors of cumulative, five-day exposure and may be an improved metric compared to hand wipes.
\end{abstract}

\footnotetext{
“Corresponding Author: Phone: 919-613-8717. Fax: 919-684-8741. heather.stapleton@ duke.edu..

Associated Content

Supporting Information

Additional information on the average PFR and metabolite levels measured in field blanks (Table S1), an expanded Spearman correlation matrix for the exposure metrics (Table S2), and the Spearman correlations based on time spent in home (Table S3) can be found in supporting information.

$\mathrm{SH}, \mathrm{KH}, \mathrm{TW}$, and HS declare no competing financial interest. KAA, an author of this research, discloses a financial interest in MyExposome, Inc., which is marketing products related to the research being reported. The terms of this arrangement have been reviewed and approved by Oregon State University in accordance with its policy on research conflicts of interest. The authors have no other disclosures.
} 


\section{Keywords}

Wristbands; hand wipes; organophosphate flame retardants; exposure; urinary metabolites

\section{Introduction}

Consumer products such as furniture, electronics, and building materials are typically treated with an array of chemicals to provide desired characteristics. In particular, flame retardants are applied to these products to reduce their flammability and adhere to national and state level fire safety standards. Polybrominated diphenyl ethers (PBDEs) were once among the most commonly applied flame retardants in consumer products; however, due to their persistence, bioaccumulation, and toxicity, the use of PBDEs has been largely phased out, leading to increased use of many alternative flame retardants. ${ }^{1,2}$ Organophosphate flame retardants (PFRs) such as tris(1,3-dichloroisopropyl) phosphate (TDCIPP), tris(1-chloro-2isopropyl) phosphate (TCIPP), and triphenyl phosphate (TPHP) are now among the most commonly used flame retardants in polyurethane foam (PUF) from upholstered furniture and have been widely detected in both air and dust from indoor environments. ${ }^{2-6}$ Components of Firemaster ${ }^{\circledR} 550$ (FM550), another frequently detected commercial flame retardant mixture that is composed of approximately $60 \%$ organophosphate compounds [e.g. TPHP and monosubstituted isopropylated triaryl phosphate (mono-ITP)] and $40 \%$ brominated compounds, have also been quantified from a number of environmental samples such as PUF from furniture and indoor dust. ${ }^{2,7,8}$

Due to their prevalence in indoor environments, human exposure to PFRs is common and their metabolites are frequently detected in human urine (Figure 1). Bis(1,3dichloroisopropyl) phosphate (BDCIPP) and diphenyl phosphate (DPHP), the respective metabolites of TDCIPP and TPHP, have been measured extensively in the general population. ${ }^{9-12}$ A hydroxylated metabolite of TCIPP, bis(1-chloro-2-isopropyl) 1hydroxy-2-propyl phosphate (BCIPHIPP), was recently identified in urine and widely detected in a large population study in Australia. ${ }^{13}$ While a specific biomarker for mono-ITP (present in FM550) has not been identified, isopropylphenyl phenyl phosphate (ip-PPP) has been suggested as a potential metabolite/biomarker. ${ }^{11}$

Toxicology studies in animals conducted with PFRs suggest that they may be associated with adverse health impacts such as carcinogenicity, cardiotoxicity, endocrine disruption, and neurotoxicity; one epidemiological study in adult men found an association between increased PFR metabolites and reduced hormone levels, specifically BDCIPP and DPHP levels in urine associated with total T3 and BDCIPP levels in urine associated with TSH levels. ${ }^{14-21}$ Accurate means of assessing personal exposure are essential to characterizing human health risks related to these flame retardants. Indoor dust has been considered an important exposure pathway, with PBDE concentrations in serum significantly and positively correlated to dust concentrations. ${ }^{22}$ However, this same relationship between indoor dust and PFR metabolites in urine has not been observed. ${ }^{23}$ While hand wipes have been reliable in demonstrating exposure to PBDEs and PFRs and have been positively associated with spot urine samples, both of these samples are limited by measurement of 
exposure at a single time point. ${ }^{12,24}$ Hand wipes are likely to primarily capture particlebound contaminants, which poses additional limitations since many flame retardants are semi-volatile and are distributed between the particle-bound and vapor phases. Urinalysis of PFR metabolites can also be challenging due to the rapid metabolism of these compounds, with half-lives estimated around several hours using animal models. ${ }^{25-27}$ To further characterize potential health outcomes, epidemiological studies require a reliable means of assessing longer-term exposures to these compounds compared to the short-term exposure measures provided by urinalysis.

Recently, silicone wristbands have been utilized as passive samplers in examining adult ambient and occupational exposures to a suite of compounds used in personal care, industrial processes, and general consumer products. ${ }^{28,29}$ These wristbands present a noninvasive and inexpensive method of quantifying personal exposures across multiple microenvironments and within a multi-day time period. However, the association between concentrations of compounds found on the wristbands and internal exposure has not been investigated. In the present study, we sought to determine the effectiveness of using silicone wristbands as an exposure tool in association with PFR metabolites in urine, and we further sought to compare wristbands to hand wipes as metrics of exposure. To our knowledge, this is the first study to validate the use of wristbands in capturing exposure to organophosphate flame retardant chemicals by measuring urinary metabolites.

\section{Materials and Methods}

\section{Study Design}

Study participants were recruited from the Duke University community and general population around Durham County, North Carolina $(n=40)$ by flyer and word of mouth in June through August of 2015. Eligible participants were at least eighteen years of age and were willing to wear wristbands for five days, to provide three urine and single hand wipe samples, and to complete questionnaires. All study protocols and related materials were approved by the Duke University Institutional Review Board, and all participants gave informed consent prior to providing any information or personal samples.

\section{Wristband Collection}

Commercially available silicone wristbands were purchased in a single size (24hourwristbands.com, Houston, TX) and cleaned using two twelve-hour Soxhlet extractions with 1:1 ethyl acetate:hexane (v/v) followed by 1:1 ethyl acetate:methanol (v/v), a method adapted from O'Connell, et. al (2014). ${ }^{28}$ After allowing the wristbands to passively dry in the fume hood, each one was wrapped in aluminum foil and placed in a labeled $40 \mathrm{~mL}$ amber jar. Participants were asked to wear their wristbands continuously for a designated five-day period through sleeping, bathing, and any other daily activities, beginning on the morning of day 1 and removing the wristband at the same time of morning on day 6. Wearing the wristband while bathing may allow for compounds sorbed to the wristbands to be washed off in a similar manner as on the skin, as well as accounting for exposures occurring during bathing (e.g. application of soap). At the designated time, participants removed the wristband, wrapped it in a new sheet of clean foil and placed it 
back in the amber jar. The wristbands were stored at $-20^{\circ} \mathrm{C}$ until extraction. Four wristbands that were not deployed were wrapped in aluminum foil and stored at room temperature to serve as field blanks with the extraction.

\section{Urine Collection}

Participants were asked to provide first morning void urine samples on three separate days during the five-day period in which they were wearing the wristbands (day 2, day 4, and day 6). Urine samples were collected in standard polypropylene specimen containers and stored at $-20^{\circ} \mathrm{C}$ until analysis. Just prior to analysis, equal volumes of the three individual urine samples were pooled to form a mixed/average sample.

\section{Questionnaires}

Study participants completed three short online questionnaires over the five days they wore the wristband (day 1, day 3, and day 5). Participants were asked to provide demographic information and to record the time spent in various microenvironments during that day (e.g. time spent in the home, work, and car). Mean fractions of days spent in these locations were calculated based on total hours in the day reported by the participant and averaged across the three questionnaires. For individuals who did not complete all three questionnaires, averages were performed based on those that were completed ( $85 \%$ completed all three questionnaires). Participants were also asked to report the average number of times that they washed their hands each day (never, 1-3, 4-6, 7-9, 10+ times per day) and the average number of times that they bathed each week (never, 1-2, 3-5, 6-8, 9-11, 12-14, 15+ times per week). The question regarding average number of times hands are washed a day was asked on two separate days, and the lower range reported was used in statistical analyses. For the purpose of later analysis, washing behaviors and time spent in the home, work, and car were dichotomized based on the median reported value (or category).

\section{Hand Wipe Collection}

Three inch square cotton twill wipes were pre-cleaned in a single twelve-hour Soxhlet extraction using 1:1 hexane:acetone (v/v) then dried in a fume hood and wrapped in aluminum foil. Hand wipe samples were collected from each participant on the morning of day 6 by gloved research personnel by soaking a pre-cleaned twill wipe with about $3 \mathrm{~mL}$ of isopropyl alcohol and wiping the entire surface area of both of the participant's hands from fingertips to wrist including between the fingers. This hand wipe collection protocol is similar to those described in previously published studies. ${ }^{12,30,31}$ The hand wipe was then rewrapped in foil and stored at $-20^{\circ} \mathrm{C}$ until analysis. Four twill wipes prepared as described above were stored wrapped in aluminum foil at room temperature to serve as field blanks.

\section{Wristband and Hand wipe Sample Extraction}

Wristband and hand wipe samples were extracted and analyzed using previously published methods for each matrix for the aforementioned organophosphate flame retardants: TDCIPP, TCIPP, TPHP, and mono-ITP. ${ }^{12,28}$ Each wristband, including the field blanks, was cut using solvent-rinsed scissors into two equal pieces to ensure that the wristbands would be submerged under the solvent within a Soxhlet apparatus used for extraction. Prior to 
beginning the extraction, the bands were spiked with $\mathrm{d}_{15}$-TDCIPP $(162 \mathrm{ng})$ and ${ }^{13} \mathrm{C}$-TPHP $(100 \mathrm{ng})$ as internal standards. The wristbands were Soxhlet extracted with 1:1 hexane:acetone (v/v) for 12 hours, and the extracts were concentrated using an automated nitrogen evaporation system (Turbo Vap II, Zymark Inc.). Extracts were filtered with a $25 \mathrm{~mm}$ syringe filter with a 0.2 micrometer PTFE membrane to remove larger particles. This concentrated extract were later cleaned using a Florisil solid-phase extraction cartridge (Supelclean ENVI-Florisil, $6 \mathrm{~mL}$, 500-mg bed weight; Supelco), eluting the F1 fraction with $10 \mathrm{~mL}$ hexane (brominated compounds) and the $\mathrm{F} 2$ fraction with $10 \mathrm{~mL}$ ethyl acetate (PFRs), which was adapted from the method developed by Van den Eede, et. al (2012). ${ }^{32}$ Using a nitrogen evaporator system, each fraction was concentrated to about $1 \mathrm{~mL}$ then transferred to an autosampler vial for gas chromatography-mass spectrometry (GC/MS) analysis (Agilent Technologies, Models 6890N and 5975, respectively). The F1 concentrated fraction was stored at $-20^{\circ} \mathrm{C}$ for future analysis. To measure recovery of the organophosphate internal standards in the wristbands, $\mathrm{d}_{9}$-tris(2-chloroethyl) phosphate ( $\mathrm{d}_{9}$ TCEP; $227 \mathrm{ng}$ ) and $\mathrm{d}_{15}$-TPHP (429 ng) were spiked into each sample to measure recovery of $\mathrm{d}_{15}$-TDCIPP and ${ }^{13} \mathrm{C}$-TPHP, respectively. Recoveries of $\mathrm{d}_{15}$-TDCIPP and ${ }^{13} \mathrm{C}$-TPHP averaged $33 \pm 5 \%$ and $99 \pm 6 \%$, respectively, in all samples. Prior to the Florisil cleanup, recovery of the $\mathrm{d}_{15}$-TDCIPP was $94 \pm 5 \%$, which suggests some of the compound were lost with additional processing. It is possible that short oligomers were extracted from the wristbands during the Soxhlet extraction which may have sorbed both labeled and unlabeled TDCIPP which were retained on the Florisil SPE column. However, due to the use of isotope-labeled standards, we still have confidence in the accuracy of our results following the cleanup step. Four lab blanks were analyzed alongside the wristbands and field blanks for quality assurance and quality controls.

The whole hand wipe samples were each spiked with $\mathrm{d}_{15}$-TDCIPP (180 ng) and ${ }^{13} \mathrm{C}$-TPHP $(50 \mathrm{ng})$ as internal standards and extracted three times via sonication with 1:1 hexane: acetone $(\mathrm{v} / \mathrm{v})$. The combined extract of roughly $45 \mathrm{~mL}$ was concentrated to $1 \mathrm{~mL}$ using a nitrogen evaporator system, then transferred to an autosampler vial for GC/MS analysis. Recoveries of the internal standards in the hand wipes were measured by spiking all samples with $\mathrm{d}_{15}$-TPHP (429 ng). Recoveries of $\mathrm{d}_{15}$-TDCIPP and ${ }^{13} \mathrm{C}$-TPHP averaged $119 \pm 14 \%$ and $97 \pm 12 \%$, respectively.

For both wristbands and hand wipes, mono-ITP was quantitated using a commercial mixture of FM550 and assuming the percent of mono-ITP in FM550 by mass is $32 \% .{ }^{8}$ All isomers at $368 \mathrm{~m} / \mathrm{z}$ were integrated over a retention time of 15.90 minutes to comprise the mass of mono-ITP in each sample. PFR concentrations in wristbands and hand wipes were blank corrected based on the average concentrations measured in the field blanks (Supplemental Table S1). Higher background levels were measured in the wristbands relative to the hand wipes for some chemicals. The Soxhlet extraction process may have contributed to the higher wristband field blank levels in wristbands; however, the wristband field blanks were still significantly lower than levels measured in the wristband samples. Method detection limits (MDLs) were calculated as three times the standard deviation of the levels in the field blanks. MDLs for the PFRs ranged from $5.7 \mathrm{ng}$ for TPHP to $30.3 \mathrm{ng}$ for TCIPP on wristbands and $0.19 \mathrm{ng}$ for TPHP to $11.21 \mathrm{ng}$ for TDCIPP on hand wipes (Table 2). 


\section{Urine Sample Processing}

Specific gravity was measured for each pooled urine sample using a digital handheld refractometer (Atago) prior to analysis. Urine samples were analyzed for BDCIPP, BCIPP, DPHP and ip-PPP following the methods described by Cooper et al (2011) and Butt et al (2014) and for BCIPHIPP using methods described by Van den Eede et al (2015). ${ }^{11,13,33}$ For each pooled sample, $5.0 \mathrm{~mL}$ of urine was spiked with internal standards $\mathrm{d}_{10}$-BDCIPP (10 ng), $\mathrm{d}_{10}$-DPHP ( $8.8 \mathrm{ng}$ ), and $\mathrm{d}_{9}$-TCEP ( $25 \mathrm{ng}$ ) and placed with $1 \mathrm{M}$ sodium acetate buffer $(\mathrm{pH} 5)$ and an enzyme solution (1000 units per $\mathrm{mL} \beta$-glucoronidase and 33 units per $\mathrm{mL}$ sulfatase activity in $0.2 \mathrm{M}$ sodium acetate buffer at $\mathrm{pH}$ 5) then incubated overnight at $37^{\circ} \mathrm{C} .{ }^{13}$ With the enzyme digestion step, the glucoronide and sulfate conjugates of BCIPHIPP cannot be differentiated from any of the free compound that may present in the urine. The five analytes (BDCIPP, BCIPP, DPHP, ip-PPP, and BCIPHIPP) were extracted via mixed-mode anion exchange solid-phase extraction and measured using atmospheric pressure chemical ionization liquid chromatography-tandem mass spectrometry (Agilent Technologies, Model 6410). ${ }^{33}$ Recoveries of the internal standards were evaluated using ${ }^{13} \mathrm{C}_{2}$-DPHP (25 ng) for $\mathrm{d}_{10}$-BDCIPP and $\mathrm{d}_{10}$-DPHP and $\mathrm{d}_{15}$-TDCIPP (25 ng) for $\mathrm{d}_{9}$ TCEP, both of which were spiked into all of the urine samples. Average recoveries for $\mathrm{d}_{10^{-}}$ BDCIPP, $\mathrm{d}_{10}$-DPHP, and $\mathrm{d}_{9}$-TCEP were $108 \pm 77 \%, 111 \pm 22 \%$, and $52 \pm 15 \%$. The MDL was calculated using three times the standard deviation of the blanks normalized to the volume of urine extracted. Lab blanks and a urine Standard Reference Material (SRM 3673; National Institute of Standards and Technology, Gaithersburg, MD) were extracted alongside the samples for quality assurance and quality control. Specific gravity-normalized measurements in SRM 3673 were $1.49 \pm 0.15 \mathrm{ng} / \mathrm{mL}, 0.32 \pm 0.01 \mathrm{ng} / \mathrm{mL}, 0.29 \pm 0.02$ $\mathrm{ng} / \mathrm{mL}$, and $3.56 \pm 0.06 \mathrm{ng} / \mathrm{mL}$ for BDCIPP, BCIPHIPP, DPHP, and ip-PPP, respectively. These values are similar to levels reported by A. Covaci's group during an interlab comparison exercise with this material (personal communication).

\section{Statistical Analyses}

All statistical analyses were performed using SAS statistical software (version 9.4; SAS Institute Inc., Cary, NC). Analyses were conducted for analytes in which the detection frequency was $>70 \%$. For concentrations below the MDL, the concentration was replaced by MDL divided by 2. Preliminary examination of the data indicated that the concentrations of PFRs and metabolites were log-normally distributed. Thus, Spearman correlation coefficients $\left(\mathrm{r}_{\mathrm{s}}\right)$ were calculated to examine the associations between the wristbands, hand wipes, and urine.

To further explore associations, linear regression models were performed to determine if any of the measured or queried variables were associated with urinary metabolite concentrations $\left(\log _{10}\right.$-transformed concentrations of analytes were used in regression analyses). Beta coefficients were exponentiated to facilitate interpretation and represented the multiplicative change in the outcome relative to the reference category or to a one-unit increase in continuous variables. PFR concentrations on wristbands and hand wipes were split into tertile categories to examine associations with the corresponding urine metabolites while reducing effects of outliers. Through all analyses, statistical results were assessed at a level of $a=0.05$ for significance. 
Specific gravity was measured in order to account for dilution of the urine samples (range of 1.0030 to 1.0254). Urine analyses of metabolites and associations with the corresponding PFRs on wristbands and hand wipes were assessed with raw metabolite concentrations and specific-gravity-corrected values. At least one pooled urine sample had extremely low specific gravity, which was unexpected for a mix of three first morning void samples. When analyses were run excluding this urine sample, the magnitude of the correlations remained unchanged. Analysis results using each set of urine concentrations were essentially not differentiable, and the specific-gravity adjusted associations and graphs are presented here.

\section{Results and Discussion}

All forty participants completed at least one of the three questionnaires with the majority (95\%) completing two or more. Approximately two-thirds of the participants were female, and the average age of participants at the time of the survey was 29.8 years (range of 20 to 60 years) (Table 1$)$.

\section{PFRs in Individual Matrices}

Wristbands-TDCIPP, TCIPP, TPHP, and mono-ITP were detected in all of the silicone wristbands (Table 2). The geometric means of TDCIPP and TCIPP were much higher than TPHP and mono-ITP on the bands. The ratio of geometric means of mono-ITP to TPHP (1.7:1) on the wristbands is similar to the relative percent compositions of these compounds in the FM550 mixture, suggesting that the TPHP concentrations on the wristbands may be at least partially attributed to exposure to FM550. ${ }^{8}$ Mono-ITP has been measured in occupational air samples and non-targeted water analyses but to our knowledge has not been previously detected and quantified in hand wipes or wristbands. ${ }^{34,35}$ A recently published study also measured PFR compounds on wristbands and verified the stability of these compounds on the wristbands through time in storage and temperature changes. ${ }^{36}$ However, levels on the wristbands could not be compared between the two studies because of the units in which PFR concentrations were reported. While dust was not collected in this study, wristband PFR concentrations in this study reflected the trend of relative geometric means in indoor dust from recent studies in central North Carolina, with TCIPP present at higher levels than TDCIPP and TPHP. ${ }^{12,23,24,32,37}$ Given that dust is an important exposure matrix and pathway, this could indicate that similar sources of these PFRs are reaching the wristbands and settling in dust particles. Additionally, dust particles could be sorbing to the wristband through physical contact or settling on the band from the air, which could explain the similar observed trend.

Hand Wipes-PFRs were detected in all of the hand wipes except for TDCIPP, which was detected in $95 \%$ of the samples (Table 2). Geometric mean amounts of mono-ITP and TDCIPP were two times higher on the hand wipes than the other two compounds, with mono-ITP being detected at levels nearly five times greater than TPHP. Although detection frequencies were slightly higher in our current cohort, concentrations of TDCIPP and TPHP on the hand wipes were similar to previously reported levels in a small cohort of adults recruited in 2012 from central North Carolina. ${ }^{12}$ TDCIPP was present at higher levels on the 
hand wipes compared to TPHP and TCIPP which reflects the previously observed trend for hand wipes but is dissimilar to the pattern typically observed in U.S. indoor dust. ${ }^{12,31}$

Specific gravity-corrected urine-The target PFR metabolites were detected in all of the urine samples except for BCIPP (18\% detection) (Table 2). Therefore, total BCIPHIPP, including glucoronide and sulfate conjugates and the free compound, was used as the primary metabolite to examine associations with TCIPP. Geometric mean concentrations of BDCIPP and DPHP were $2.23 \mathrm{ng} / \mathrm{mL}$ and $1.14 \mathrm{ng} / \mathrm{mL}$, respectively. Concentrations of DPHP were similar to previously reported levels but BDCIPP within this cohort seemed to be two to three times higher than other reported studies of U.S. adult cohorts, ${ }^{12,33}$ with levels most comparable to adult women over age eighteen who were sampled in Butt et. al (2014). ${ }^{11}$ The difference in BDCIPP concentration observed in our cohort may reflect higher exposure to TDCIPP, timing and location of the urine sample, or other lifestyle or demographic differences within the cohort. As a hypothesized metabolite of mono-ITP, ipPPP was measured at two times higher concentrations than previously detected in adults, which could suggest increased exposure to FM550 components. ${ }^{11}$ Our work is the first to measure concentrations of BCIPHIPP in a U.S. population; however, concentrations were roughly similar to an Australian cohort in which it was first measured. ${ }^{13}$

\section{Comparing Wristbands and Hand Wipes to Urine}

The levels of TDCIPP and TCIPP on wristbands were significantly and positively correlated with their corresponding urinary metabolites, BDCIPP and BCIPHIPP $\left[\mathrm{r}_{\mathrm{s}}=0.59, \mathrm{p}<0.0001\right.$, and $r_{s}=0.62$, $p<0.0001$, respectively (Table 3, Figure 2)], suggesting that silicone wristbands worn over a five day period do capture personal exposures. TPHP and mono-ITP concentrations on wristbands were not correlated with DPHP. DPHP may be a urinary metabolite of these two parent compounds; however, other chemicals may also metabolize to form DPHP [e.g. 2-ethylhexyl diphenyl phosphate (EHDPP), isodecyl diphenyl phosphate (id-DPP)] which may explain the lack of an association. ${ }^{38}$ Similarly, mono-ITP concentrations were not correlated with ip-PPP, as ip-PPP may not be the primary metabolite or may be one of several potential metabolites of mono-ITP. Exposures to TPHP could also originate from diet or other sources such as nail polish and plastic bottles, which were not expected to be captured by the wristbands but would still result in the presence of urinary DPHP. ${ }^{39,40}$

Concentrations of TDCIPP and TCIPP on the hand wipes were also positively associated with urinary metabolites (i.e. BDCIPP and BCIPHIPP), although the correlations were smaller in magnitude than those for wristbands and urinary metabolites (Table 3 ). The correlation between hand wipe TDCIPP and BDCIPP in urine was similar to a previous study with a North Carolina adult cohort, which is generally smaller in magnitude than the hand wipe to serum correlations observed in children for PBDEs. ${ }^{12,41}$ This finding suggests that wristbands are likely better metrics of integrated exposure to these two compounds over five days compared to the use of hand wipes. DPHP levels in urine were not strongly correlated with TPHP and mono-ITP on hand wipes, again suggesting other potential sources of exposure to DPHP. ${ }^{40,42}$ On the wristbands, mono-ITP was positively associated with urinary BDCIPP $\left(\mathrm{r}_{\mathrm{s}}=0.33, \mathrm{p}=0.04\right)$ which may reflect co-exposure with the parent 
compound, TDCIPP, particularly as the levels of mono-ITP and TDCIPP on the wristband were also weakly correlated $\left(r_{s}=0.29, p=0.06\right)$ (Table 3, Supplemental Table S2). Within each matrix (hand wipes or wristbands), mono-ITP and TPHP levels were highly correlated $\left(r_{s}=0.71\right.$ and $r_{s}=0.77$, respectively, $p<0.0001$ for both), which could indicate that the two compounds were co-occurring and may share similar sources. PFRs in wristbands and hand wipes were significantly associated between matrices for TDCIPP and TCIPP $\left(r_{s}=0.39\right.$ and $\mathrm{r}_{\mathrm{s}}=0.69$, respectively). Correlations for each PFR within a matrix (hand wipes or wristbands) can be found in Supporting Information.

While hand wipes are thought to capture information about multiple microenvironment in the short-term from recent exposures, wristbands may be capturing information from multiple microenvironments over the course of several days. ${ }^{30,31}$ Different exposure pathways may be captured from the hand wipes compared to the wristbands. Hand wipes are more likely to contain exposures from contact with surfaces while wristbands may better reflect exposures from the gas phase and airborne particulates. Since hand wipes were collected either at home or when participants were coming from home, the hand wipes were more likely to reflect these short-term exposures in the home environment. To further investigate this possibility, we calculated correlations between PFRs and metabolites restricting to participants that spent more time at home (e.g. more time in a single microenvironment). TDCIPP and TCIPP on hand wipes and urinary metabolite concentrations were more strongly correlated when we restricted to participants who reported spending more time in their home $\left(n=18 ; r_{s}=0.72\right.$ and $r_{s}=0.67 ; p<0.01$ for both; see Supplemental Table S3). Although the correlation between TDCIPP in wristbands was also slightly higher in participants who reported spending more time in their home environment $\left(r_{s}=0.84\right)$, the correlation between TCIPP in wristbands and urinary BCIPHIPP was similar regardless of the percentage of time spend in different environments. This finding may indicate that wristbands perform better for these compounds because they integrate information over a longer time course and from more diverse microenvironments than hand wipes taken at a single point in time. However, hand wipes may be the better measure for examining exposures from surface contact or absorption from the dermal exposure pathway as described by Weschler and Nazaroff (2012), as well as from any hand-to-mouth activity. ${ }^{43}$ The dermal absorption pathway, particularly for PFRs, should be considered since models may be underestimating absorbed dosages, and handwashing may significantly alter absorption. ${ }^{44}$

Linear regression analyses were also conducted to examine exposure measures categorized by tertiles and personal characteristics as predictors of urinary metabolites. These analyses were conducted with and without outliers, particularly the one individual with notably low BCIPHIPP levels. Exclusion of the outliers did not change the interpretation of the results; therefore, we reported the outcomes including outliers. On average, concentrations of the three PFR metabolites were significantly higher in participants who had wristbands or hand wipes containing higher levels of TDCIPP, TCIPP, and TPHP (Table 4). For instance, participants with the highest levels of TDCIPP on their wristbands had urinary concentration of BDCIPP 3.34 times those with the lowest levels of TDCIPP $\left(10^{\beta}=3.34 ; 95 \%\right.$ Confidence Interval (CI): 1.70, 6.56; $\mathrm{p}=0.0009$ ). 
With a few notable exceptions, behavior and activity patterns as well as age and sex were generally not associated with measured concentrations of PFRs on wristbands (Table 5). However, higher average time spent in a car, categorized dichotomously by the median (1 hour), was significantly associated with higher BDCIPP levels $\left(10^{\beta}=2.33 ; 95 \%\right.$ CI: 1.31 , 4.17) (Table 5). More frequent washing behaviors such as more daily handwashing and weekly baths or showers, also categorized dichotomously by median, were associated with higher levels of BCIPHIPP in urine $\left(10^{\beta}=2.61 ; 95 \%\right.$ CI: 1.07, 6.31). BCIPHIPP was recently identified as a metabolite of TCIPP, and it is uncertain whether other sources related to washing may be contributing to BCIPHIPP levels in urine or other participant behaviors may be confounding this result. ${ }^{45}$

Our results should be interpreted in the context of several limitations. Only one paired wristband and hand wipe were collected per participant which does not account for individual variability. Further studies are needed to examine the utility of wristbands over different periods of time (i.e. 1 day, 5 days, 10 days). In this study, we selected 5 days as the time period, but it is uncertain whether this is representative of average, longer-term exposures which could occur over the course of weeks, months, or even years. Wristband samples were collected over a short period of time (June to August) in central North Carolina so it is unclear how generalizable these results are to other seasons and climates. Our sample size of forty was relatively small which limited the number of variables that we could examine using multivariate regression analyses and may have restricted the power to detect other meaningful associations in the data. Additionally, first morning void urine samples were collected which should serve as a reliable average urine sample; however, collecting all urine samples throughout the five-day period would have served as improved measure for total exposure to the measured compounds. In our analyses, we assume urine PFR metabolites as the gold standard for exposure measurements for our comparisons with wristbands and hand wipes although there are still many data gaps in understanding PFR metabolism and excretion through urine. Lastly, our sample population included adults generally from the local community surrounding Duke University which led to a relatively homogenous cohort. This may limit the generalizability of our results to the U.S. adult population but does not alter the internal validity of our study.

Overall, our results suggest that wristbands may serve as an additional and improved metric of exposure for organophosphate flame retardants compared to hand wipes. This is demonstrated by the greater magnitudes of correlations observed for TDCIPP and TCIPP with their urinary metabolites compared to those in hand wipes. These PFRs are classified as semi-volatile organic compounds, which suggests that they are likely partitioning between the gaseous and solid phases and specifically sorbing to multiple surfaces. The volatile fraction of compounds may also be a large source of exposure and uptake which may be underestimated with current exposure metrics. While hand wipes serve as more of a crosssectional, single-time point sample, wristbands may provide some insight into average exposures across a longer time period and capture some of the inhalation exposure that is currently lacking in measurements. Additionally, with the ability to capture exposures to multiple compounds simultaneously, silicone wristbands can improve how we examine exposures to mixtures in the environment. It is currently unclear whether the concentrations of these PFRs, particularly TDCIPP and TCIPP, are sorbing to the wristbands from the 
gaseous phase or from particles, which could be contributing to exposure via inhalation, ingestion of aerosolized small particles, or slow dermal absorption. Future studies should aim to elucidate the sources of TDCIPP and TCIPP on the wristbands and thereby obtain a greater understanding of the primary pathway by which people are being exposed to these compounds.

\section{Supplementary Material}

Refer to Web version on PubMed Central for supplementary material.

\section{Acknowledgements}

Funding for this research was provided by the NIEHS (Grant R01ES016099) and the EPA (Grant 83564201). We also gratefully acknowledge the effort provided by our research participants.

\section{References}

(1). Rahman F, Langford KH, Scrimshaw MD, Lester JN. Polybrominated diphenyl ether (PBDE) flame retardants. Sci. Total Environ. 2001; 275(1-3):1-17. [PubMed: 11482396]

(2). Stapleton HM, Sharma S, Getzinger G, Ferguson PL, Gabriel M, Webster TF, Blum A. Novel and high volume use flame retardants in US couches reflective of the 2005 PentaBDE phase out. Environ. Sci. Technol. 2012; 46(24):13432-13439. [PubMed: 23186002]

(3). Bergh C, Torgrip R, Emenius G, Ostman C. Organophosphate and phthalate esters in air and settled dust - a multi-location indoor study. Indoor Air. 2011; 21(1):67-76. [PubMed: 21054550]

(4). Stapleton HM, Klosterhaus S, Eagle S, Fuh J, Meeker JD, Blum A, Webster TF. Detection of organophosphate flame retardants in furniture foam and U.S. house dust. Environ. Sci. Technol. 2009; 43(19):7490-7495. [PubMed: 19848166]

(5). Marklund A, Andersson B, Haglund P. Screening of organophosphorus compounds and their distribution in various indoor environments. Chemosphere. 2003; 53(9):1137-1146. [PubMed: 14512118]

(6). Van den Eede N, Dirtu AC, Neels H, Covaci A. Analytical developments and preliminary assessment of human exposure to organophosphate flame retardants from indoor dust. Environ. Int. 2011; 37(2):454-461. [PubMed: 21176966]

(7). Stapleton HM, Allen JG, Kelly SM, Konstantinov A, Klosterhaus S, Watkins D, Mcclean MD, Webster TF. Alternate and new brominated flame retardants detected in U.S. house dust. Environ. Sci. Technol. 2008; 42(18):6910-6916. [PubMed: 18853808]

(8). Klosterhaus, S.; Konstantinov, A.; Davis, E.; Klein, J. Characterization of organophosphorus chemicals in a PentaBDE replacement mixture and their detection in biosolids. 2009.

(9). Carignan CC, McClean MD, Cooper EM, Watkins DJ, Fraser AJ, Heiger-Bernays W, Stapleton HM, Webster TF. Predictors of tris(1,3-dichloro-2-propyl) phosphate metabolite in the urine of office workers. Environ. Int. 2013; 55:56-61. [PubMed: 23523854]

(10). Stapleton HM, Klosterhaus S, Keller A, Ferguson PL, van Bergen S, Cooper E, Webster TF, Blum A. Identification of flame retardants in polyurethane foam collected from baby products. Environ. Sci. Technol. 2011; 45(12):5323-5331. [PubMed: 21591615]

(11). Butt CM, Congleton J, Hoffman K, Fang M, Stapleton HM. Metabolites of organophosphate flame retardants and 2-ethylhexyl tetrabromobenzoate in urine from paired mothers and toddlers. Environ. Sci. Technol. 2014; 48(17):10432-10438. [PubMed: 25090580]

(12). Hoffman K, Garantziotis S, Birnbaum LS, Stapleton HM. Monitoring indoor exposure to organophosphate flame retardants: hand wipes and house dust. Environ. Health Perspect. 2015; 123(2):160-165. [PubMed: 25343780]

(13). Van den Eede N, Heffernan AL, Aylward LL, Hobson P, Neels H, Mueller JF, Covaci A. Age as a determinant of phosphate flame retardant exposure of the Australian population and identification of novel urinary PFR metabolites. Environ. Int. 2015; 74:1-8. [PubMed: 25277340] 
(14). OEHHA Reproductive and Cancer Hazard Assessment Branch. Evidence on the Carcinogenicity of Tris(1,3-dichloro-2-propyl) phosphate. 2011.

(15). McGee SP, Konstantinov A, Stapleton HM, Volz DC. Aryl phosphate esters within a major PentaBDE replacement product induce cardiotoxicity in developing zebrafish embryos: potential role of the aryl hydrocarbon receptor. Toxicol. Sci. 2013; 133(1):144-156. [PubMed: 23377616]

(16). Gerlach CV, Das SR, Volz DC, Bisson WH, Kolluri SK, Tanguay RL. Mono-substituted isopropylated triaryl phosphate, a major component of Firemaster 550, is an AHR agonist that exhibits AHR-independent cardiotoxicity in zebrafish. Aquat. Toxicol. 2014; 154:71-79. [PubMed: 24865613]

(17). Dishaw LV; Powers CM, Ryde IT, Roberts SC, Seidler FJ, Slotkin T. a, Stapleton HM. Is the PentaBDE replacement, tris (1,3-dichloro-2-propyl) phosphate (TDCPP), a developmental neurotoxicant? Studies in PC12 cells. Toxicol. Appl. Pharmacol. 2011; 256(3):281-289. [PubMed: 21255595]

(18). Belcher SM, Cookman CJ, Patisaul HB, Stapleton HM. In vitro assessment of human nuclear hormone receptor activity and cytotoxicity of the flame retardant mixture FM 550 and its triarylphosphate and brominated components. Toxicol. Lett. 2014; 228(2):93-102. [PubMed: 24786373]

(19). Pillai HK, Fang M, Beglov D, Kozakov D, Vajda S, Stapleton HM, Webster TF, Schlezinger JJ. Ligand binding and activation of PPAR $\gamma$ by Firemaster® 550: effects on adipogenesis and osteogenesis in vitro. Environ. Health Perspect. 2014; 122(11):1225-1232. [PubMed: 25062436]

(20). Noyes PD, Haggard DE, Gonnerman GD, Tanguay RL. Advanced Morphological -- Behavioral Test Platform Reveals Neurodevelopmental Defects in Embryonic Zebrafish Exposed to Comprehensive Suite of Halogenated and Organophosphate Flame Retardants. Toxicol. Sci. 2015; 145(1):177-195. [PubMed: 25711236]

(21). Meeker JD, Cooper EM, Stapleton HM, Hauser R. Exploratory analysis of urinary metabolites of phosphorus-containing flame retardants in relation to markers of male reproductive health. Endocr. Disruptors. 2013; 1(1):e26306.

(22). Johnson PI, Stapleton HM, Sjodin A, Meeker JD. Relationships between Polybrominated Diphenyl Ether Concentrations in House Dust and Serum. Environ. Sci. Technol. 2010; 44(14): 5627-5632. [PubMed: 20521814]

(23). Dodson RE, Van den Eede N, Covaci A, Perovich LJ, Brody JG, Rudel RA. Urinary Biomonitoring of Phosphate Flame Retardants: Levels in California Adults and Recommendations for Future Studies. Environ. Sci. Technol. 2014; 48(23):13625-13633. [PubMed: 25388620]

(24). Stapleton HM, Misenheimer J, Hoffman K, Webster TF. Flame retardant associations between children's handwipes and house dust. Chemosphere. 2014; 116:54-60. [PubMed: 24485814]

(25). Nomeir AA, Kato S, Matthews HB. The metabolism and disposition of tris(1,3-dichloro-2propyl) phosphate (Fyrol FR-2) in the rat. Toxicol. Appl. Pharmacol. 1981; 57(3):401-413. [PubMed: 7222047]

(26). Sasaki K, Suzuki T, Takeda M, Uchiyama M. Metabolism of phosphoric acid triesters by rat liver homogenate. Bull. Environ. Contam. Toxicol. 1984; 33(3):281-288. [PubMed: 6478075]

(27). Lynn RK, Wong K, Garvie-Gould C, Kennish JM. Disposition of the flame retardant, tris(1,3dichloro-2-propyl) phosphate, in the rat. Drug Metab. Dispos. 1981; 9(5):434-441. [PubMed: 6117442]

(28). O’Connell SG, Kincl LD, Anderson KA. Silicone wristbands as personal passive samplers. Environ. Sci. Technol. 2014; 48(6):3327-3335. [PubMed: 24548134]

(29). O'Connell SG, McCartney MA, Paulik LB, Allan SE, Tidwell LG, Wilson G, Anderson KA. Improvements in pollutant monitoring: Optimizing silicone for co-deployment with polyethylene passive sampling devices. Environ. Pollut. 2014; 193:71-78. [PubMed: 25009960]

(30). Stapleton HM, Kelly SM, Allen JG, Mcclean MD, Webster TF. Measurement of polybrominated diphenyl ethers on hand wipes: Estimating exposure from hand-to-mouth contact. Environ. Sci. Technol. 2008; 42(9):3329-3334. [PubMed: 18522114]

(31). Stapleton HM, Misenheimer J, Hoffman K, Webster TF. Flame retardant associations between children's handwipes and house dust. Chemosphere. 2014 
(32). Van den Eede N, Dirtu AC, Ali N, Neels H, Covaci A. Multi-residue method for the determination of brominated and organophosphate flame retardants in indoor dust. Talanta. 2012; 89:292-300. [PubMed: 22284495]

(33). Cooper EM, Covaci A, van Nuijs ALN, Webster TF, Stapleton HM. Analysis of the flame retardant metabolites bis(1,3-dichloro-2-propyl) phosphate (BDCPP) and diphenyl phosphate (DPP) in urine using liquid chromatography-tandem mass spectrometry. Anal. Bioanal. Chem. 2011; 401(7):2123-2132. [PubMed: 21830137]

(34). Sjödin A, Carlsson H, Thuresson K, Sjölin S, Bergman Å, Östman C. Flame Retardants in Indoor Air at an Electronics Recycling Plant and at Other Work Environments. Environ. Sci. Technol. 2001; 35(3):448-454. [PubMed: 11351713]

(35). Allan IJ, Harman C, Ranneklev SB, Thomas KV, Grung M. Passive sampling for target and nontarget analyses of moderately polar and nonpolar substances in water. Environ. Toxicol. Chem. 2013; 32(8):1718-1726. [PubMed: 23625759]

(36). Kile ML, Scott RP, O’Connell SG, Lipscomb S, MacDonald M, McClelland M, Anderson KA. Using silicone wristbands to evaluate preschool children's exposure to flame retardants. Environ. Res. 2016; 147:365-372. [PubMed: 26945619]

(37). Cequier E, Ionas AC, Covaci A, Marcé RM, Becher G, Thomsen C. Occurrence of a Broad Range of Legacy and Emerging Flame Retardants in Indoor Environments in Norway. Environ. Sci. Technol. 2014; 48(12):6827-6835. [PubMed: 24846325]

(38). Nishimaki-Mogami T, Minegishi K, Tanaka A, Sato M. Isolation and identification of metabolites of 2-ethylhexyl diphenyl phosphate in rats. Arch. Toxicol. 1988; 61(4):259-264. [PubMed: 3377680]

(39). Bittner GD, Yang CZ, Stoner MA. Estrogenic chemicals often leach from BPA-free plastic products that are replacements for BPA-containing polycarbonate products. Environ. Heal. 2014; 13(1):41.

(40). Mendelsohn E, Hagopian A, Hoffman K, Butt CM, Lorenzo A, Congleton J, Webster TF, Stapleton HM. Nail polish as a source of exposure to triphenyl phosphate. Environ. Int. 2016; 86:45-51. [PubMed: 26485058]

(41). Stapleton HM, Eagle S, Sjödin A, Webster TF. Serum PBDEs in a North Carolina toddler cohort: Associations with handwipes, house dust, and socioeconomic variables. Environ. Health Perspect. 2012; 120(7):1049-1054. [PubMed: 22763040]

(42). van der Veen I, de Boer J. Phosphorus flame retardants: properties, production, environmental occurrence, toxicity and analysis. Chemosphere. 2012; 88(10):1119-1153. [PubMed: 22537891]

(43). Weschler CJ, Nazaroff WW. SVOC exposure indoors: fresh look at dermal pathways. Indoor Air. 2012; 22(5):356-377. [PubMed: 22313149]

(44). Abou-Elwafa Abdallah M, Pawar G, Harrad S. Human dermal absorption of chlorinated organophosphate flame retardants; implications for human exposure. Toxicol. Appl. Pharmacol. 2016; 291:28-37. [PubMed: 26712466]

(45). Van den Eede N, Maho W, Erratico C, Neels H, Covaci A. First insights in the metabolism of phosphate flame retardants and plasticizers using human liver fractions. Toxicol. Lett. 2013; 223(1):9-15. [PubMed: 23994729] 


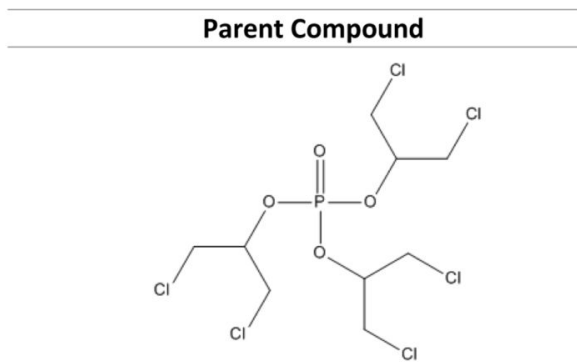

Tris(1,3-dichloroisopropyl) phosphate (TDCIPP)

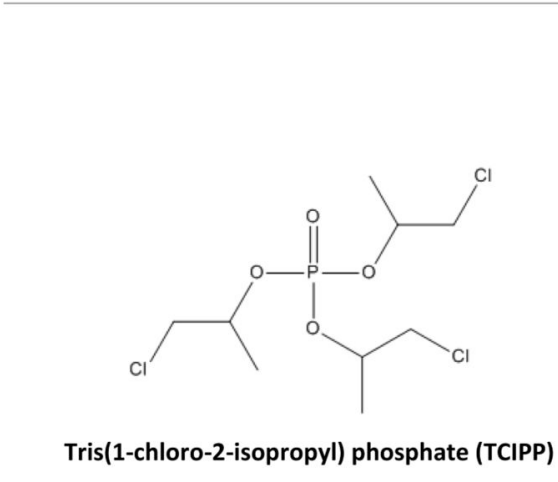

Tris(1-chloro-2-isopropyl) phosphate (TCIPP)
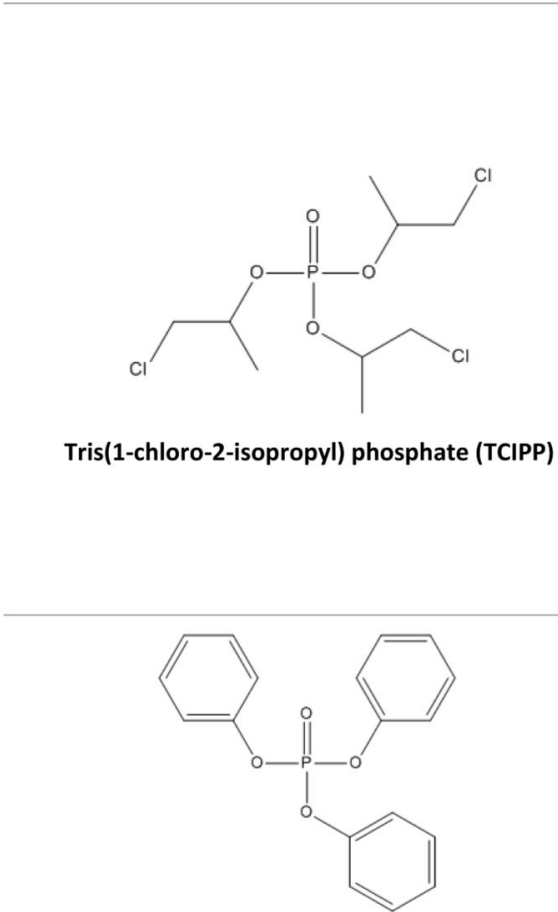

Triphenyl phosphate (TPHP)

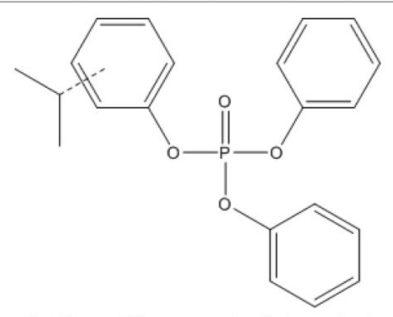
(mono-ITP)

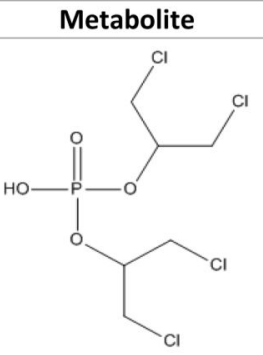

Bis(1,3-dichloroisopropyl) phosphate (BDCIPP)

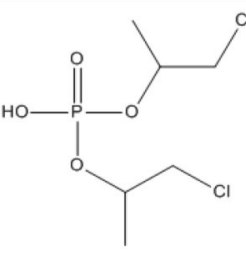

Bis(1-chloro-2-isopropyl) phosphate (BCIPP)

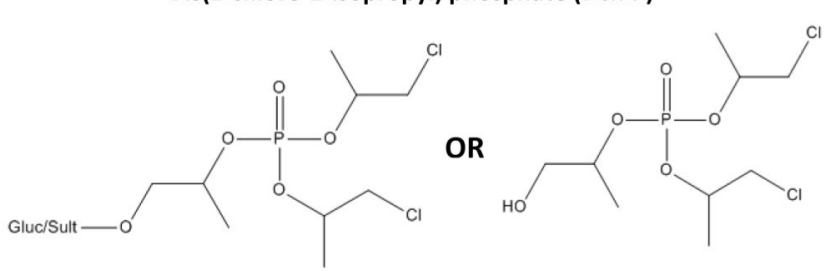

Bis(1-chloro-2-isopropyl) 1-hydroxy-2-isopropyl phosphate (BCIPHIPP)*

Mono-substituted isopropyl triphenyl phosphate

Figure 1. Parent compound and urinary metabolites for common organophosphate flame retardants. Starred metabolites $(*)$ are considered the primary metabolites for analysis 

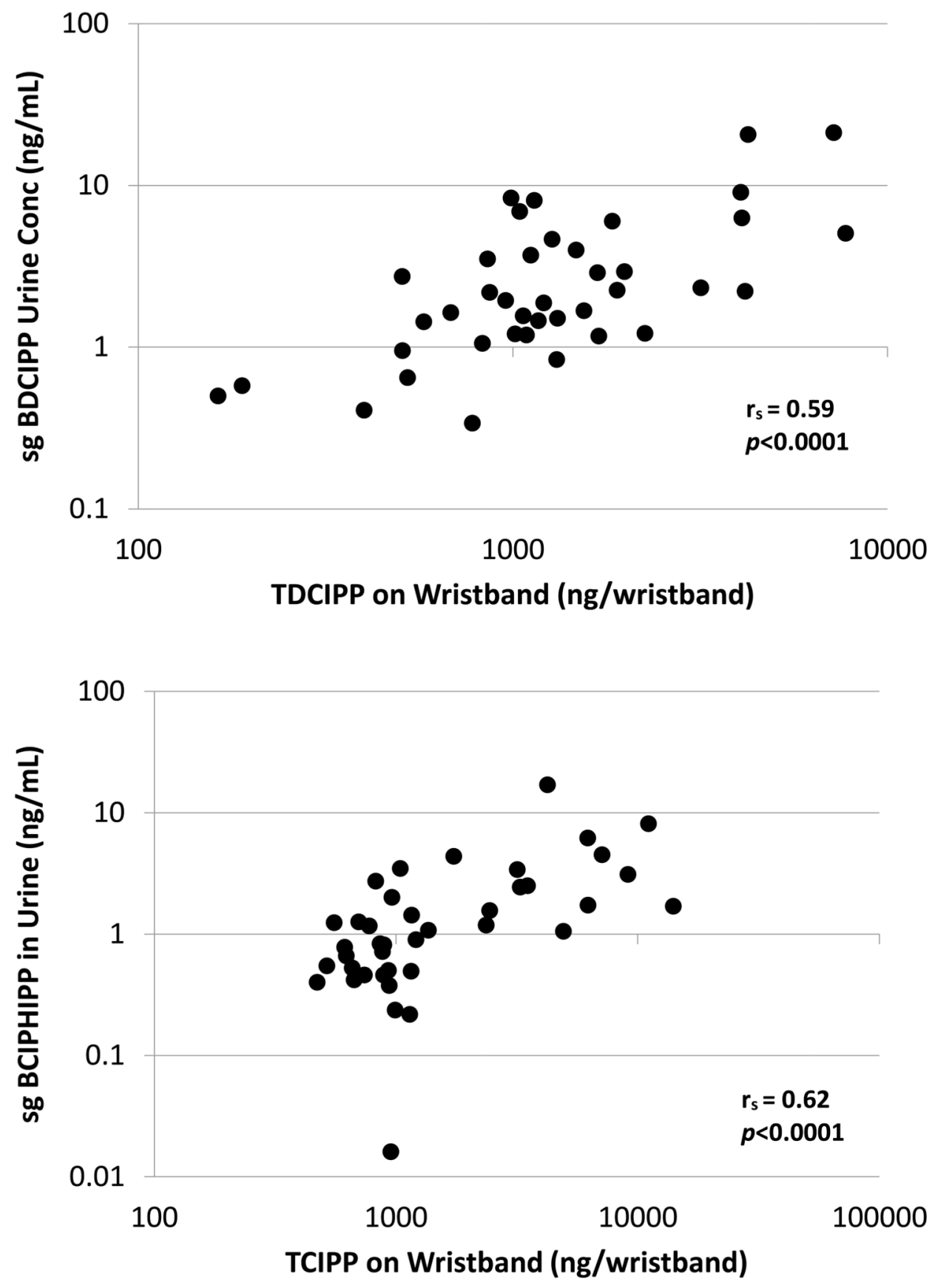

Figure 2. Correlation of TDCIPP and TCIPP on wristbands with their urinary metabolites 


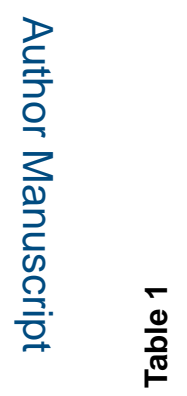

로을

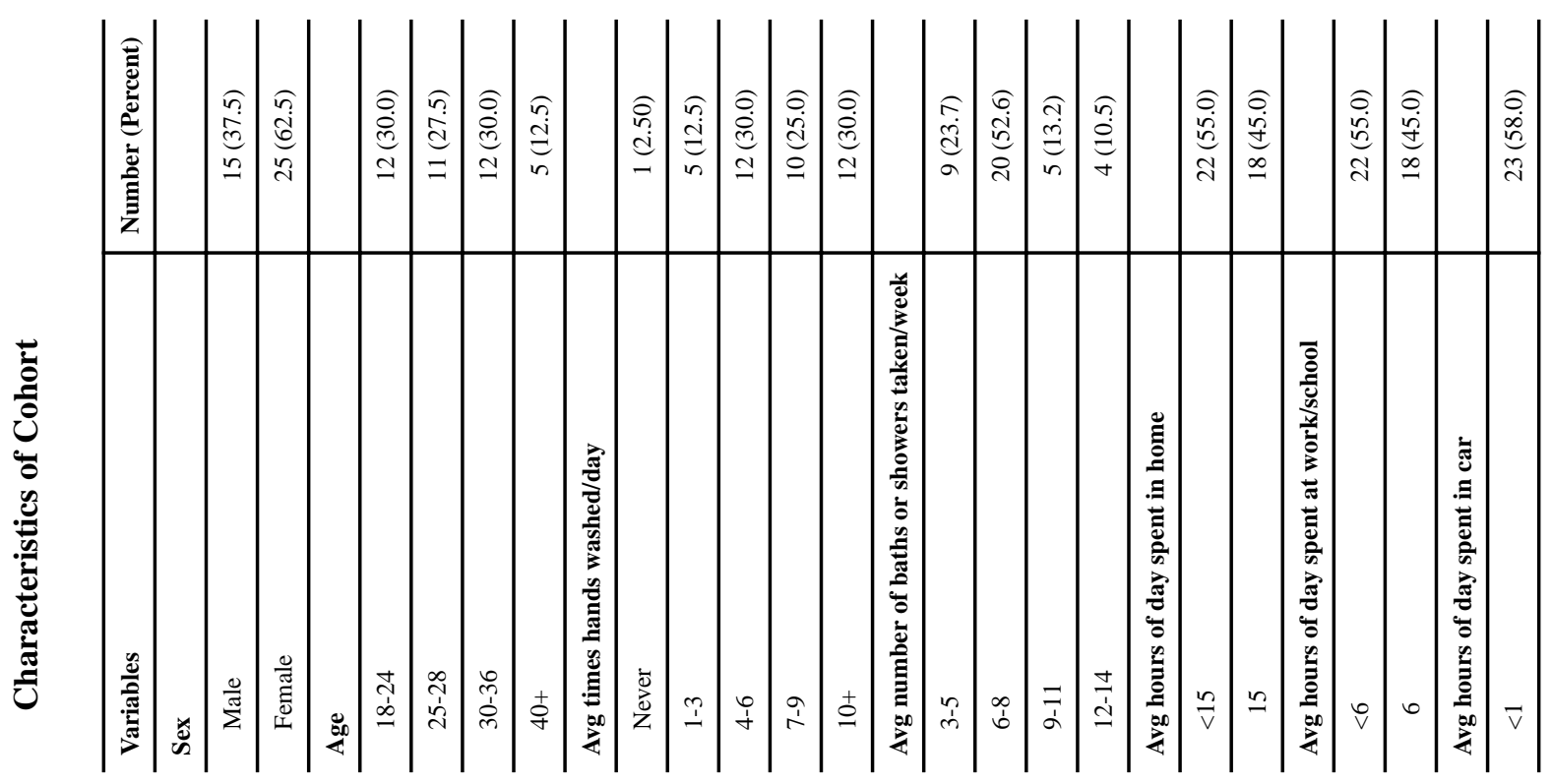

Environ Sci Technol. Author manuscript; available in PMC 2017 April 19. 


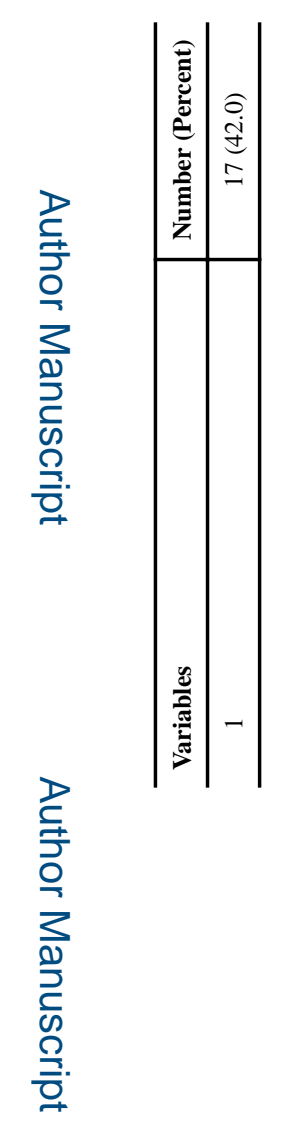

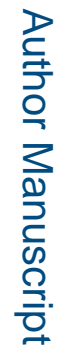

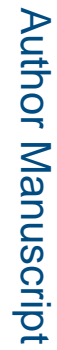

Environ Sci Technol. Author manuscript; available in PMC 2017 April 19. 


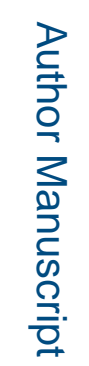

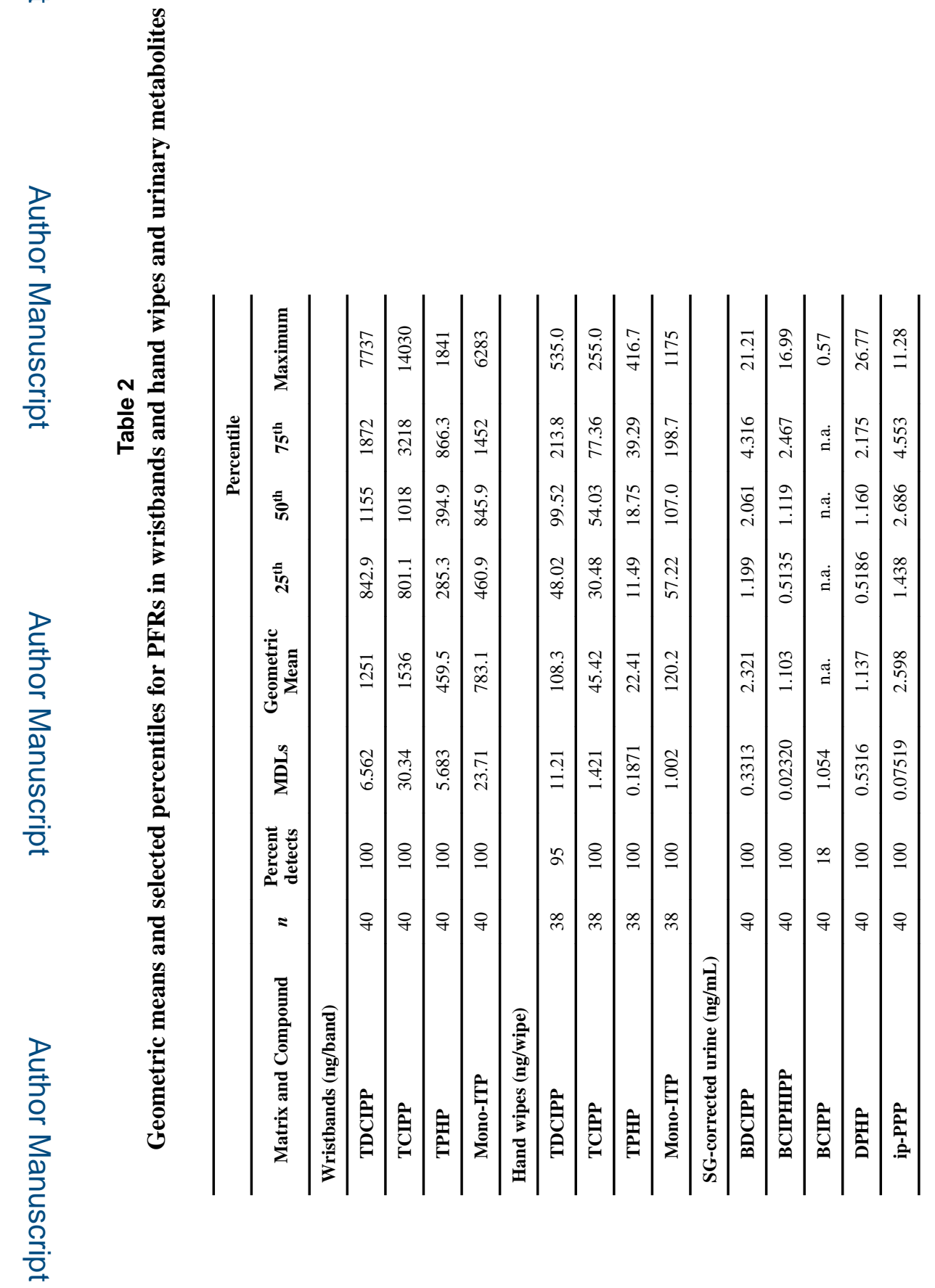

Environ Sci Technol. Author manuscript; available in PMC 2017 April 19. 


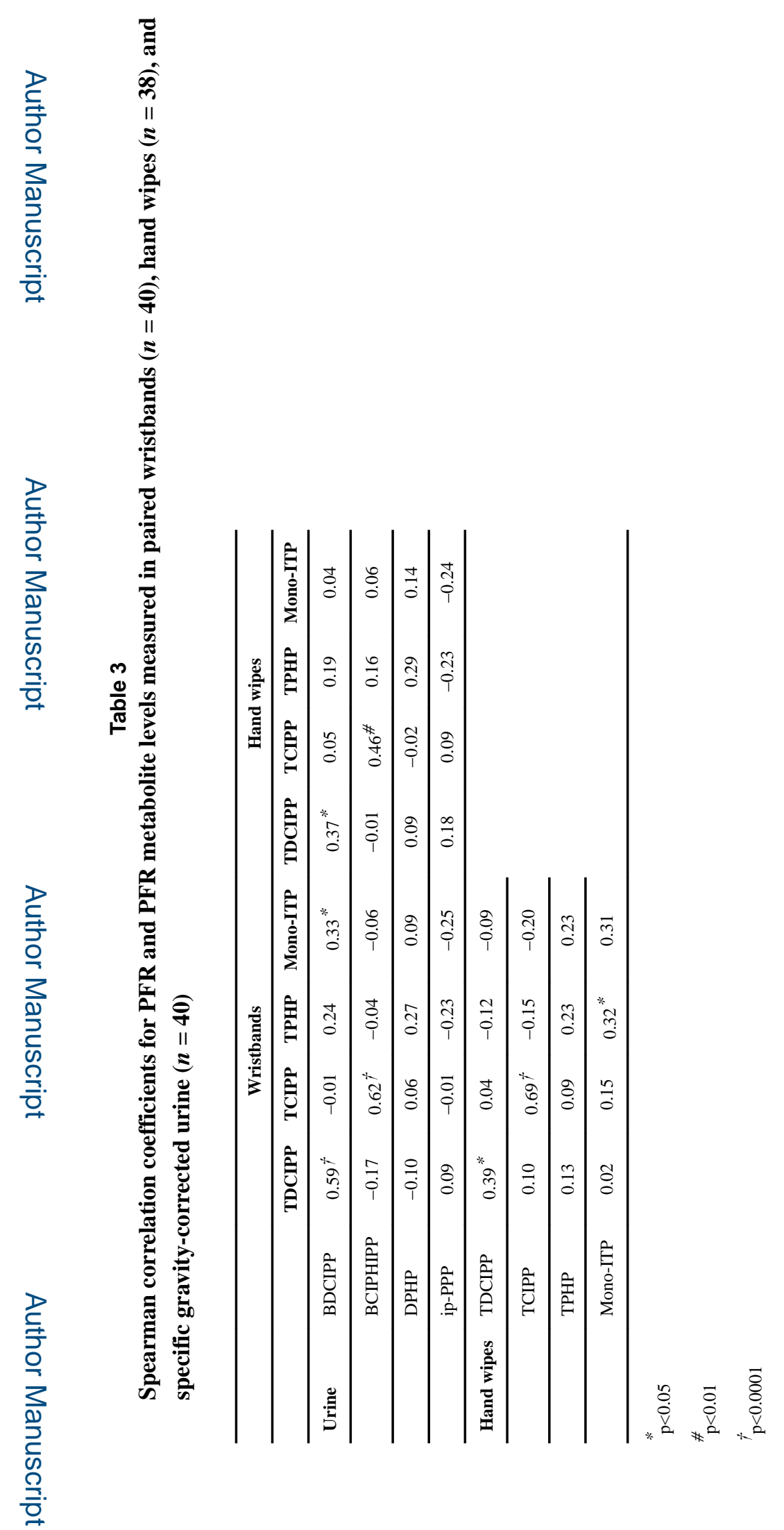




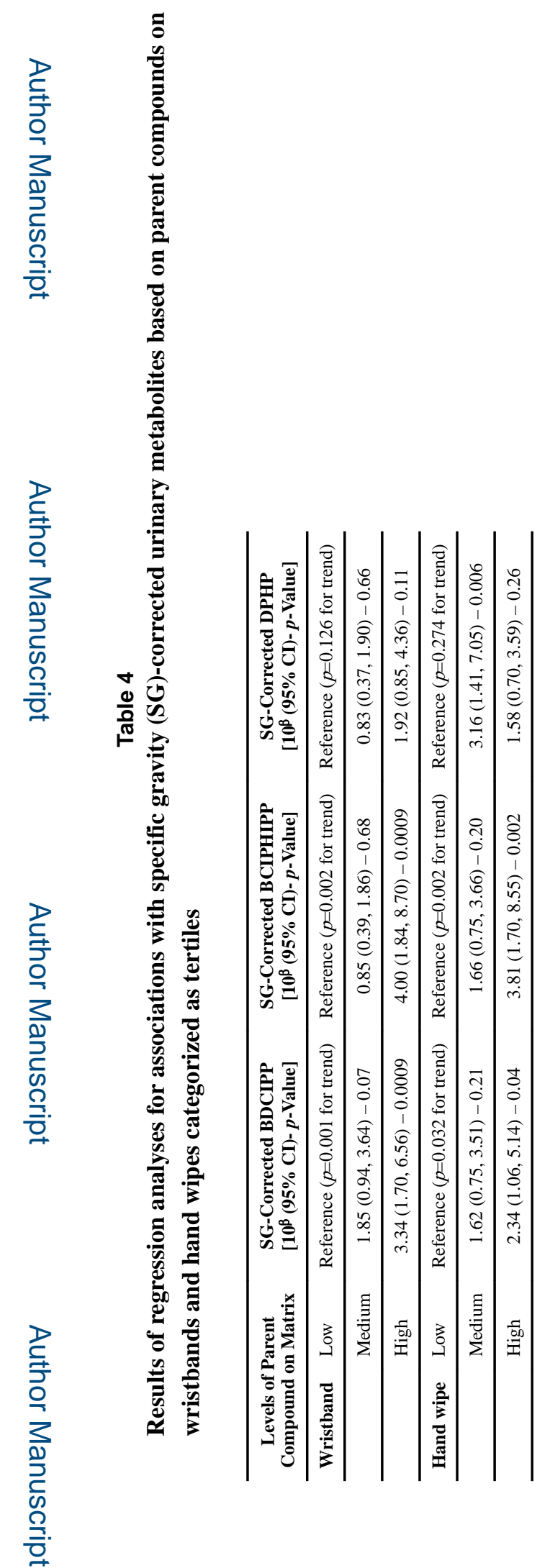

Environ Sci Technol. Author manuscript; available in PMC 2017 April 19. 


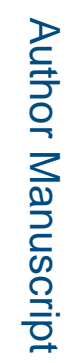

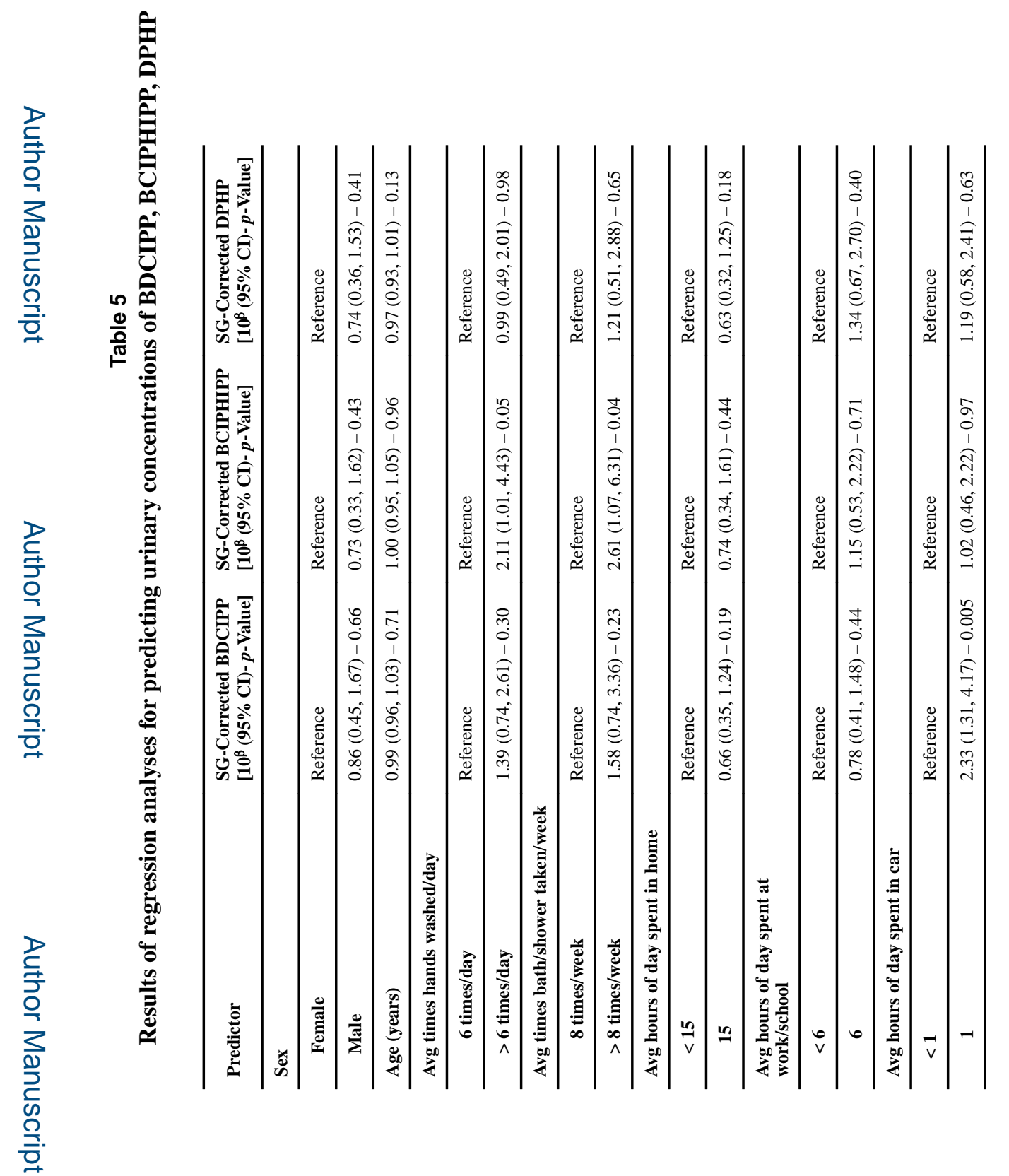

Environ Sci Technol. Author manuscript; available in PMC 2017 April 19. 